\title{
EFECTO DE LAS BOTAS DE BOMBERO Y LAS PLANTILLAS VISCOELÁSTICAS SOBRE LA FUERZA DE IMPACTO DE LA COMPONENTE VERTICAL DE LA FUERZA DE REACCIÓN DEL SUELO
}

\author{
Jesús Cámara-Tobalina \\ Facultad de Ciencias del Deporte de la Universidad del País Vasco / Euskal Herriko \\ Unibertsitatea. \\ jesus.camara@ehu.es
}

RESUMEN

\begin{abstract}
Objetivos: determinar el efecto de las botas de bombero sobre la componente vertical de la fuerza de reacción del suelo (FRS), en el contacto inicial del talón en el suelo o también denominada fuerza de impacto, así como analizar el efecto de las plantillas viscoelásticas implantadas en las botas de bombero sobre esta fuerza durante la marcha. Metodología: Se registró la magnitud de la fuerza de impacto (FZI) de la componente vertical de la FRS, el tiempo hasta la producción de esta fuerza (TZI) y su gradiente de carga (GC); 39 bomberos sin ningún tipo de patología durante los dos años previos a la toma de registros formaron parte del estudio. Se comparó la marcha en tres condiciones diferentes de calzado: 1) la marcha con botas de bombero, 2) la marcha con botas de bombero a las cuales se les había implantado unas plantillas viscoelásticas y 3) la marcha con calzado deportivo.

Resultados: mostraron una mayor producción, así como una mayor magnitud de la fuerza de impacto con botas de bombero respecto a la marcha con calzado deportivo (13,1 vs. 2,6 \% de producción de la fuerza de impacto y 61,39 \pm 35,18\% PC (peso corporal) vs. 49,38 \pm 22,99\% PC, respectivamente). La marcha con plantillas viscoelásticas implantadas en las botas de bombero no mostró diferencias significativas en ningún parámetro que caracteriza la fuerza de impacto respecto a la marcha sin plantillas. Conclusiones: los resultados de este estudio muestran por un lado, una menor amortiguación de la fuerza de impacto con las botas de bombero en comparación con la marcha con calzado deportivo y por otro, la ineficacia de las plantillas viscoelásticas implantadas en las botas de bombero para la mejora de la amortiguación de la fuerza de impacto durante la marcha a velocidad espontánea.
\end{abstract}

\section{EFFECT OF FIREFIGHTER BOOTS AND VISCOELASTIC INSOLES ON THE IMPACT FORCE OF THE VERTICAL COMPONENT OF THE GROUND REACTION FORCE}

\begin{abstract}
The present study was aimed at determining the effect of firefighter boots on the vertical component of the ground reaction force (GRF) at heel strike, also known as heel strike transient, as well as analyzing the effect of viscoelastic insoles in firefighter boots on the heel strike during gait. The magnitude of the impact force (FZI) of the vertical component of the GRF, the time to the production of this force (TZI) and the loading rate (GC) were registered. A total of 39 firefighters with no pathologies 2 years prior to the study were recruited. Gait was compared under three different walking conditions: 1) gait with firefighter boots, 2) gait with firefighter boots and viscoelastic insoles and 3) gait with sport shoes. Results showed higher production and magnitude of the impact force during gait with firefighter boots than with sport shoes (13.1 vs. 2.6\% occurrence of the impact force and $61.39 \pm 35.18 \% \mathrm{BW}$ (bodyweight) vs. $49.38 \pm 22.99 \%$ BW, respectively). Gait with viscoelastic insoles in firefighter boots showed no significant differences in any of the parameters characterizing the impact force compared to gait without insoles. The results of this study show lower cushioning of the impact force during gait with firefighter boots in comparison to gait with sport shoes and the inefficiency of the viscoelastic insoles in firefighter boots to improve cushioning of the impact force at a natural walking speed.
\end{abstract}

KEYWORDS: cushioning, insoles, biomechanics. 


\section{INTRODUCCIÓN}

Desde que Radin y cols. (Radin, Paul, \& Rose, 1972) establecieron la tesis de que la componente vertical de la fuerza de reacción del suelo (FRS) en el contacto inicial del talón en el suelo o también denominada fuerza de impacto es una de las causas de la degeneración articular, el interés por la amortiguación de esta fuerza se vio incrementado (Light, 1979). Durante la marcha estos impactos se producen durante el apoyo del talón en el suelo y se observa como un pico en la componente vertical de la fuerza de reacción del suelo (VFRS), cuya aparición es previa a la máxima fuerza producida durante el apoyo del peso del sujeto sobre una sola pierna (Perry, 1992) (Figura 1). La aparición de este pico, denominado fuerza de impacto (Windle, Gregory, \& Dixon, 1999), se ha relacionado con la artrosis, fascitis plantar, tendinitis, fracturas por estrés, dolores en la parte baja de la espalda e incluso dolores de cabeza (Gill \& O’Connor, 2003b; Lafortune, Lake, \& Hennig, 1996; A. Voloshin \& Wosk, 1982).

El cuerpo humano dispone de mecanismos de amortiguación de la fuerza de impacto, entre los que se encuentra la activación muscular previa al apoyo del pie en el suelo (Nigg, Cole, \& Bruggemann, 1995; Wakeling, Liphardt, \& Nigg, 2003; Wakeling, Tscharner, Nigg, \& Stergiou, 2001): el tibial anterior y el cuádriceps han mostrado ser eficaces en la amortiguación de esta fuerza (Jefferson, Collins, Whittle, Radin, \& O'Connor, 1990; Lafortune, et al., 1996; Murray, Kory, Clarkson, \& Sepic, 1966; Perry, 1992; Sánchez Lacuesta et al., 1999). Por otro lado, la almohadilla del talón, formada por una masa flexible de tejido adiposo con un grosor comprendido entre 13 y 21mm. (Steinbach \& Russel, 1964), distribuye la fuerza durante el apoyo del talón (Wang, Shau, Hsu, Chen, \& Chien, 1999) amortiguando de esta forma también el impacto (Bennett \& Ker, 1990; Jorgensen \& Bojsen-Moller, 1989; Jorgensen \& Ekstrand, 1988; Wang, et ál., 1999). Esta almohadilla ha demostrado aumentar su capacidad amortiguadora mediante su confinamiento (Jorgensen \& Ekstrand, 1988; Lafortune \& Henning, 1992).

A pesar de los mecanismos de amortiguación de los que dispone el cuerpo humano, estos no son suficientes para amortiguar totalmente la fuerza de impacto (Folman, Wosk, Voloshin, \& Liberty, 1986), lo que provocaría en la VFRS una desaparición de esta fuerza (Verdini et ál., 2000). Debido a ello, una de las funciones del calzado es proporcionar una protección adicional frente al impacto del talón en el suelo (Aguinaldo, Litavish, \& Morales, 2002; Bates, 1984; Cámara \& Gavilanes, 2005; Carmichael \& Whittle, 1999; Lafortune \& Henning, 1992; Light, MacLellan, \& Klenerman, 1980; Pratt, Rees, \& Rodgers, 1986; Verdini, et al., 2000; A. S. Voloshin \& Wosk, 1981; Michael W. Whittle, 1999).

Las características amortiguadoras de diferentes tipos de calzado tales como el de cuero (Menz, Latt, Tiedemann, Mun San Kwan, \& Lord, 2004; Menz, Lord, \& Fitzpatrick, 2003; Wakeling, et ál., 2003), el calzado deportivo (Hull, Brewer, \& Hawkins, 1995; Keller et al., 1996), las botas de montaña (Hettinga, Stefanyshin, Fairburn, \& Worobets, 2005) y las botas militares (Evans, 1982; Milgrom et ál., 1985) han sido analizadas. No obstante, no hay estudios que analicen las características amortiguadoras de las botas de bombero, a pesar de estar sujetas a una normativa de seguridad (TC 94/SC 3) (ISO, 2007) que podría comprometer la amortiguación de la fuerza de impacto. Dicha normativa, establecida por el Comité Europeo para la Estandarización (CEN) con la colaboración del Comité Técnico de Seguridad Personal de la Organización Internacional para la Estandarización menciona, entre otras condiciones, que las botas de bombero deben soportar una compresión de $15 \mathrm{kN}$ y un impacto de 200J. Esto implica que la suela de las botas de bombero esté fabricada con materiales duros que podrían no favorecer la amortiguación de la fuerza de impacto y que por lo tanto, la implantación de plantillas viscoelásticas en las botas podría proporcionar una amortiguación adicional. No obstante, no se ha encontrado ningún estudio que analice la influencia de las botas de bombero sobre la fuerza de impacto durante la marcha. 
El objetivo del presente estudio es determinar el efecto de las botas de bombero sobre la fuerza de impacto durante la marcha. Además, se analizará el efecto de un tipo de plantillas viscoelásticas implantadas en las botas de bombero sobre esta fuerza.

\section{METODOLOGÍA}

Sujetos. 39 bomberos (edad $=42 \pm 5,3$ años; altura $=174,5 \pm 4,6 \mathrm{~cm}$; y masa $=78 \pm 14,4 \mathrm{~kg}$ ) tomaron parte en el estudio tras ser informados de forma oral y escrita de las características de la presente investigación y firmar el preceptivo consentimiento informado. Ningún bombero tenía una patología en el miembro inferior durante al menos dos años previos a la toma de registros. El estudio se realizó siguiendo el código ético de la declaración de Helsinki.

Instrumentos. Se utilizó una plataforma de fuerzas (Dinascan/IBV, 8.2, Instituto de Biomecánica de Valencia, España) embebida en el suelo con una frecuencia de $500 \mathrm{~Hz}$. Para comprobar que la marcha de los sujetos estaba dentro de los valores de normalidad se utilizó el programa NedAMH/IBV 2.1 (Instituto de Biomecánica de Valencia, España). La medición del tiempo que los sujetos tardaban en recorrer $11 \mathrm{~m}$ del pasillo de marcha se realizó mediante unas células fotoeléctricas (Dinascan/IBV, Instituto de Biomecánica de Valencia, España).

Procedimientos. Los registros se realizaron en tres condiciones de marcha: con las botas de bombero Elten Sichezheitsschule Scharz (C) (condición I), con las plantillas viscoelásticas Sorbothane (C) implantadas en las botas de bombero (condición II), y con calzado deportivo (condición III). Este último consistió en el que cada bombero normalmente utilizaba para hacer ejercicio físico. En todos los casos este calzado disponía de un sistema de amortiguación. Los sujetos dispusieron de 3 minutos para familiarizarse con cada condición de calzado. Este tiempo se considera suficiente para evitar el efecto arrastre (Payne, Zammitt, \& Patience, 2005). Este efecto consiste en que los sujetos, al realizar los registros en nueva condición de marcha, pueden estar influenciados por la condición anterior (Pardo Merino \& Ruiz Díaz, 2002).

El orden de registro entre las condiciones de calzado se realizó de forma aleatoria (Esenyel, Walden, Gitter, Walsh, \& Karacan, 2004; Oeffinger et al., 1999). Previamente a la realización de los registros, los sujetos pasaban andando una vez por la plataforma de fuerzas para determinar que la marcha de cada sujeto estaba dentro de los valores de normalidad. Un solo registro se ha mostrado suficiente para analizar el grado de normalidad de los sujetos (IBV, 2004).

La línea de salida se situó al inicio de la zona de aceleración en el sentido de progresión y se modificaba dependiendo del patrón de marcha de cada sujeto en cada condición de calzado, con el objeto de que cada sujeto pisara sobre la plataforma de fuerza con el pie derecho sin variar su patrón de marcha. A los sujetos se les dio la orden de que anduvieran con la mirada al frente y sin fijarse en el emplazamiento de la plataforma. Se realizaron cinco registros válidos por cada condición de marcha. Este número de registros ha sido empleado también por otros autores (Cavanagh, Williams, \& Clark, 1979; Lake \& Robinson, 2005; Martin \& Marsh, 1992; Mills \& Barrett, 2001; Pollo, Gowling, \& Jackson, 1999; Sloss, 2002; Tilbury-Davis \& Hooper, 1999; Wearing, Urry, \& Smeathers, 2000). Se consideraba un registro válido aquel en el que los sujetos anduvieron los 15 metros del pasillo de marcha y donde el pie derecho entraba completamente en la plataforma de fuerzas sin que hubiera una modificación del patrón de marcha. Los dos metros iniciales y finales no se tuvieron en cuenta para el cálculo de la velocidad de marcha, ya que consistían respectivamente en la zona de aceleración y desaceleración, con el objeto de que se midiera la velocidad de los sujetos cuando ésta era constante. 
En cada registro se determinó la presencia o ausencia de la fuerza de impacto. De esta forma se obtuvo el porcentaje de producción de la fuerza de impacto teniendo en cuenta el número de veces que se producía dicha fuerza en los cinco registros realizados en cada condición de calzado. En el caso en el que ésta se hubiera producido se determinó la magnitud de la fuerza de impacto (FZI) y el tiempo hasta su producción (TZI) (Figura 1). FZI se normalizó con el peso corporal (PC) de los sujetos y TZI se normalizó con el tiempo de apoyo del pie sobre la plataforma de fuerza (TA) para comparar el tiempo hasta la producción de la fuerza de impacto entre las distintas condiciones de calzado. El gradiente de carga (GC) se obtuvo mediante la siguiente fórmula:

GC $\left(\right.$ PC $\left.\cdot \mathrm{s}^{-1}\right)=$ FZI (\%PC) /TZI (s)(Keller, et al., 1996)

La velocidad de los sujetos se calculó teniendo en cuenta el tiempo que invertían en recorrer 11 m del pasillo de marcha.

Figura 1. Componente vertical de la fuerza de reacción del suelo

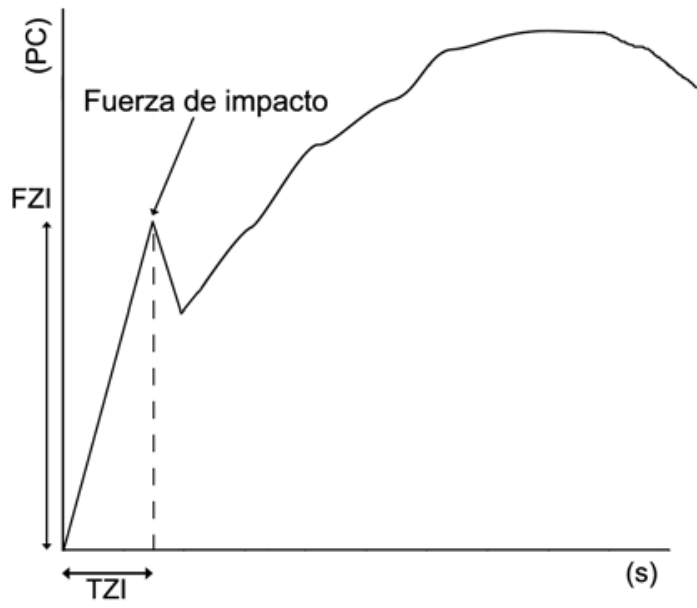

PC: peso corporal, FZI: magnitud de la fuerza de impacto, TZI: tiempo hasta la producción de la fuerza de impacto.

Análisis estadístico. Los datos se presentan como media \pm desviación estándar (DS). En los casos de cumplimiento del supuesto de normalidad se ha utilizado el ANOVA de medidas repetidas. Este análisis estadístico pone a prueba la hipótesis nula $(H 0)$ de igualdad de medias entre las distintas condiciones de marcha. La hipótesis de esfericidad se ha analizado mediante la prueba de esfericidad de Mauchly. En caso de cumplimiento de este supuesto se ha utilizado el estadístico $\mathrm{F}$ univariado en su versión esfericidad asumida para el estudio de la hipótesis nula del ANOVA. Cuando se ha rechazado la hipótesis de esfericidad se ha fundamentado la hipótesis de igualdad de medias en las estimaciones épsilon Huynh-Feldt. Cuando no se ha cumplido el supuesto de normalidad se ha realizado la prueba de Friedman para verificar o rechazar la hipótesis nula de igualdad de medias. Tanto en el análisis paramétrico como en el no paramétrico se ha realizado el análisis post hoc de Bonferroni con el objeto de determinar qué condiciones de marcha diferían entre sí. El criterio estadístico de significación fue de p<0,05. El análisis estadístico se realizó mediante el programa SPSS 19.0 (SPSS Inc., Chicago, Il, EUA).

\section{RESULTADOS}

Todos los bomberos mostraron un grado de normalidad de la marcha superior al 70\% según el programa NedAMH/IBV 2.1. Este valor está considerado dentro del rango de valores de las personas que no tienen ningún tipo de anormalidad de la marcha. La fuerza de impacto no se produjo en todas las condiciones de calzado, siendo la condición I en la que en menos ocasiones se produjo (Figura 2). 
Figura 2. Porcentaje de producción de la fuerza de impacto en las distintas condiciones.

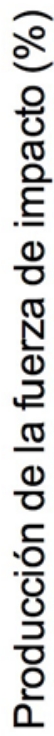

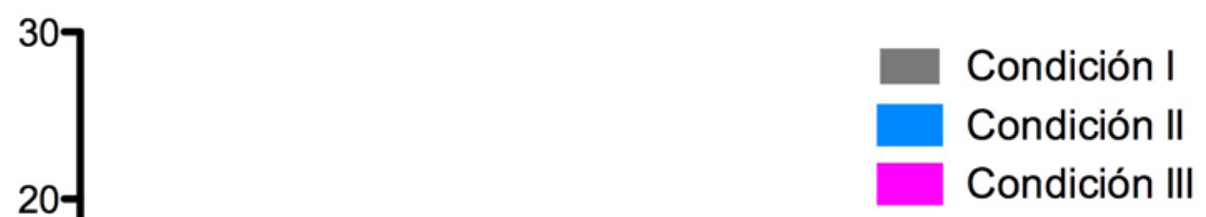

Condición I: botas de bombero Elten Sichezheitsschule Scharz (C), Condición II: botas de bombero y plantillas viscoelásticas Sorbothane @e, Condición III: calzado deportivo.

Tabla 1.

Estadísticos descriptivos de los parámetros que caracterizan la fuerza de impacto.

\begin{tabular}{lrrrrrr}
\hline & \multicolumn{2}{c}{ Condición I } & \multicolumn{2}{c}{ Condición II } & \multicolumn{2}{c}{ Condición III } \\
Parámetros & \multicolumn{1}{c}{ Media \pm DS } & Rango & \multicolumn{1}{c}{ Media \pm DS } & Rango & Media \pm DS & Rango \\
\hline FZI (\%PC) & $61,93 \pm 35,18^{*}$ & 49,76 & $58,53 \pm 30,67 \dagger$ & 68,81 & $49,38 \pm 22,99 * \dagger$ & 61,91 \\
TZI (\%TA) & $10,13 \pm 5,12$ & 7,25 & $11,93 \pm 5,33$ & 14,55 & $7,49 \pm 3,01$ & 8,61 \\
GC (\%PC· $\left.\mathbf{s}^{-1}\right)$ & $7,85 \pm 6,41$ & 10,48 & $6,40 \pm 5,59$ & 12,09 & $8,55 \pm 3,51$ & 5,84 \\
\hline
\end{tabular}

Condición I: botas de bombero Elten Sichezheitsschule Scharz (C), Condición II: botas de bombero y plantillas viscoelásticas Sorbothane (C), Condición III: calzado deportivo, DS: desviación estándar, FZI: magnitud de la fuerza de impacto, PC: peso corporal, TZI: tiempo hasta la producción de la fuerza de impacto, TA: tiempo de apoyo, GC: gradiente de carga, * diferencias significativas entre la condición III y la I para $\mathrm{p}<0,05$, $†$ diferencias significativas entre la condición III y la II para p<0,05.

Tabla 2.

Estadísticos descriptivos de los parámetros espacio-temporales.

\begin{tabular}{|c|c|c|c|c|c|c|}
\hline \multirow[b]{2}{*}{ Parámetros } & \multicolumn{2}{|c|}{ Condición I } & \multicolumn{2}{|c|}{ Condición II } & \multicolumn{2}{|c|}{ Condición III } \\
\hline & Media \pm DS & Rango & Media \pm DS & Rango & Media \pm DS & Rango \\
\hline $\mathbf{V}\left(\mathbf{m} \cdot \mathbf{s}^{-1}\right)$ & $1,36 \pm 0,10^{*}$ & 0,57 & $1,37 \pm 0,10^{*}$ & 0,53 & $1,41 \pm 0,10^{*}$ & 0,54 \\
\hline $\mathrm{CP}\left(\right.$ pasos$\left.\cdot \mathrm{min}^{-1}\right)$ & $107,40 \pm 7,31 *$ & 36,50 & $107,31 \pm 7,26 *$ & 34,30 & $111,43 \pm 7,27 *$ & 33,60 \\
\hline LP (m) & $0,76 \pm 0,04$ & 0,04 & $0,76 \pm 0,04$ & 0,17 & $0,75 \pm 0,04$ & 0,19 \\
\hline
\end{tabular}


Condición I: botas de bombero Elten Sichezheitsschule Scharz (C), Condición II: botas de bombero y plantillas viscoelásticas Sorbothane (C), Condición III: calzado deportivo, DS: desviación estándar, V: velocidad, CP: cadencia de pasos, LP: longitud de paso, * diferencias significativas entre la condición I y II con la condición III para p<0,05.

\section{DISCUSIÓN}

Éste es el primer estudio que analiza la influencia de las botas de bombero sobre la fuerza de impacto de la VFRS. Los resultados han mostrado que no sólo el porcentaje de producción de la fuerza de impacto es mayor con la marcha botas de bombero en comparación con la marcha con calzado deportivo, sino que además FZI es también mayor. Por otro lado, se ha observado que las plantillas viscoelásticas implantadas en las botas de bombero no han disminuido el impacto del talón en el suelo. Tanto con las botas de bombero así como con el calzado deportivo la fuerza de impacto se produjo por el apoyo del talón en el suelo.

En el presente estudio se ha observado un incremento significativo del porcentaje de producción de la fuerza de impacto durante la marcha con botas de bombero respecto a la marcha con calzado deportivo (Figura 2). Durante la práctica deportiva, el cuerpo humano está sometido a impactos de una gran magnitud (Lequesne, Dang, \& Lane, 1997). Además, en deportes donde los saltos son muy frecuentes, tales como el balonmano, voleibol y baloncesto se ha observado un mayor número de lesiones en el miembro inferior (Lequesne, et al., 1997; Vrezas, Elsner, Bolm-Audorff, Abolmaali, \& Seidler, 2010). Esto ha conllevado a que en el diseño del calzado deportivo se preste especial atención a la disminución de la fuerza de impacto. Esto explicaría el mayor porcentaje de producción de la fuerza de impacto durante la marcha con botas de bombero. La ausencia obtenida en el presente estudio de la fuerza de impacto con el calzado deportivo $(97,4 \%)$ concuerda con los datos obtenidos en anteriores investigaciones (McCaw, Heil, \& Hamill, 2000; Verdini, et al., 2000).

No obstante, no sólo es mayor la producción de la fuerza de impacto con botas de bombero respecto a la marcha con calzado deportivo, sino que además, en las ocasiones en las que se ha producido la fuerza de impacto, ésta presenta una significativamente mayor magnitud de FZI (Tabla 1). Sin embargo, TZI así como GC no han presentado diferencias estadísticamente significativas entre ambos tipos de calzado. Estos resultados son más llamativos al analizar la velocidad de marcha en ambas condiciones. Ésta fue, al igual que en otros estudios (Chao, Laughman, Schneider, \& Stauffer, 1983; Gill \& O’Connor, 2003a; M.W. Whittle, 1997), la adoptada espontáneamente por los bomberos, debido a que la imposición de una velocidad específica de marcha podría conllevar una modificación del patrón de marcha natural de los sujetos (Perry, 1992). Como consecuencia de ello, la marcha con botas de bombero con y sin las plantillas viscoelásticas mostró una velocidad y cadencia de pasos significativamente menor que la marcha con calzado deportivo (Tabla I). La longitud de paso por el contrario no mostró diferencias significativas entre las distintas condiciones de calzado. Teniendo en cuenta que la velocidad de marcha muestra una relación directa con la magnitud de VFRS (Keller, et al., 1996), no era esperado obtener una mayor magnitud de FZI con las botas de bombero. No obstante, la dureza de los materiales para la confección de las botas de bombero con el objeto de cumplir la normativa TC 94/SC 3, así como la ausencia de un sistema de amortiguación en las botas han podido influir en la mayor magnitud de FZI, a pesar de la menor velocidad de marcha.

Teniendo en cuenta que éste es el primer estudio donde se analiza la influencia de las botas de bombero sobre la fuerza de impacto, no se pueden comparar estos resultados con los obtenidos en anteriores estudios en un calzado similar. No obstante, la comparación con los resultados obtenidos en un calzado de aparente similitud con las botas de bombero como son las botas militares, muestra una menor magnitud de FZI con este último tipo de botas (botas de bombero: 0,61 \%PC vs. botas militares: 0,37 \%PC), sugiriendo una mayor amortiguación con las botas 
militares (Cavanagh, et al., 1979). El escaso número de sujetos que tomaron parte del estudio, así como la menor velocidad de marcha utilizada por los militares y las diferencias entre las botas de bombero y las botas militares han podido tener una influencia en la diferencia de la magnitud de FZI. Con otro tipo de calzado, como son los zapatos de cuero, también se ha obtenido una menor magnitud de FZI (35,1 \%PC) (Shiba et al., 1995). Se apunta también a los diferentes materiales y diseños entre las botas de bombero y las zapatos de cuero como posibles causas de las diferencias entre ambos estudios de la magnitud de la fuerza de impacto.

Por otro lado, cabe mencionar que a pesar de las características que se les atribuye a las plantillas viscoelásticas Sorbothane @ , su implantación en las botas de bombero con el objeto de amortiguar el impacto la fuerza de impacto, no ha originado ni un decremento en el porcentaje de producción de la fuerza de impacto ni una disminución de su magnitud y de su gradiente de carga. Estas plantillas han sido confeccionadas con un polímero viscoelástico de $4 \mathrm{~mm}$ que ha demostrado amortiguar el impacto del talón en el suelo (Folman, Wosk, Shabat, \& Gepstein, 2004). No obstante, los resultados del presente estudio cuestionan la utilidad de las plantillas Sorbothane (c) implantadas en las botas de bombero Elten Sichezheitsschule Scharz (c) para amortiguar el impacto del talón en el suelo. La dureza de la planta de la bota, con el objeto de cumplir la normativa de seguridad TC 94/SC 3, ha podido influir en la eficacia de estas plantillas para amortiguar la fuerza de impacto.

\section{CONCLUSIONES}

La mayor producción de la fuerza de impacto, así como la mayor magnitud de esta fuerza durante la marcha con botas de bombero respecto a la macha con calzado deportivo sugieren que las botas de bombero amortiguan el impacto del talón en el suelo durante la marcha a velocidad espontánea en menor medida que el calzado deportivo. La ausencia de un efecto sobre la fuerza de impacto de las plantillas viscoelásticas implantadas en las botas de bombero cuestiona la eficacia de estas plantillas para amortiguar el impacto del talón en el suelo. Se apunta a la dinámica inversa, facilitada por un sistema de fotogrametría sincronizado con el registro de la fuerza de reacción del suelo, como una metodología para profundizar en el efecto de las diferentes condiciones de calzado sobre las estructuras músculo-esqueléticas.

\section{REFERENCIAS}

Aguinaldo, A., Litavish, M., \& Morales, A. (2002). Comparison of transient force attenuation between three types of heel cushions used in athletic footwear. Gait \& Posture, 16(s1), 100-101.

Bates, B. T. (1984, September 17-19). Proceedings of the 37th Annual Conference on Engineering in Medicine and Biology. Paper presented at the Overview: The evaluaton and effetcs of heel strike, Los Ángeles, California.

Bennett, M. B., \& Ker, R. F. (1990). The mechanical properties of the human subcalcaneal fat pad in compression. Journal of Anatomy, 171(3), 131-138.

Carmichael, \& Whittle, M. W. (1999). Gender differences in the heelstrike transient. Gait \& Posture(9), 144-145.

Cavanagh, P. R., Williams, K. R., \& Clark, T. E. (1979). A comparison of ground reaction forces during walking barefoot and in shoes. In A. Morecki, K. Fidelus, K. Kedzior \& A. Wit (Eds.), Biomechanics VII-B (pp. 151-156). Baltimore: University Park Press. 
URL www.una.ac.cr/mhsalud

Chao, E. Y., Laughman, R. K., Schneider, E., \& Stauffer, R. N. (1983). Normative data of knee joint motion and ground reaction forces in adult level walking. Journal of Biomechanics, 16(3), 219-233.

Esenyel, M., Walden, G., Gitter, A., Walsh, N. E., \& Karacan, I. (2004). Gait characteristics with and without shoes. Türkiye Fiziksel Tip ve Rehabilitasyon Dergisi., 50(2), 33-37.

Evans, G. W. (1982). Stress fractures at Commando Trainning Centre Royal Marines, Lymspotine - A retrospective survey (september 1979 - october 1981). Journal of the Royal Naval Medical Service, 68, 77-81.

Folman, Y., Wosk, A., Voloshin, A., \& Liberty, S. (1986). Cyclic impacts on heel strike: a possible biomechanical factor in the etiology of degenerative disease of the human locomotor system. Archives of Orthopaedic and Trauma Surgery, 104, 363-365.

Folman, Y., Wosk, J., Shabat, S., \& Gepstein, R. (2004). Attenuation of spinal transients at heel strike using viscoelastic heel insoles: an in vivo study. Preventive Medicine, 39(2), 351354.

Gill, H. S., \& O'Connor, J. J. (2003a). Heelstrike and the pathomechanics of osteoarthrosis: a pilot gait study. Journal of Biomechanics(36), 1625-1631.

Gill, H. S., \& O'Connor, J. J. (2003b). Heelstrike and the pathomechanics of osteoarthrosis: a simulation study. Journal of Biomechanics(36), 1617-1624.

Goble, D. J., Marino, G. W., \& Potvin, J. R. (2003). The Influence of Horizontal Velocity on Interlimb Symmetry in Normal Walking. Human Movement Science, 22, 271-283.

Hettinga, B. A., Stefanyshin, D., Fairburn, J. C., \& Worobets, J. T. (2005). The 7th Symposium on Footwear Biomechanics. Paper presented at the Biomechanical effects of hiking on a non-uniform surface, Cleveland.

Hreljac, A., \& Marshall, R. N. (2000). Algorithms to determine event timing during normal walking using kinematic data. Journal of Biomechanics, 33, 783-786.

Hull, M. L., Brewer, R., \& Hawkins, D. (1995). A New Force Plate Design Incorporating Octagonal Strain Rings. Journal of Applied Biomechanics, 11, 311-321.

IBV. (2004). NedAMH/IBV Análisis de la Marcha Humana Manual de usuario. Versión 2.1. Valencia: Instituto de Biomecánica de Valencia.

ISO, ISO 20345:2004/Amd 1:2007 (ISO, 2007)

Jefferson, R. J., Collins, J. J., Whittle, M. W., Radin, E. L., \& O'Connor, J. (1990). The role of the quadriceps in controlling impulsive forces around heel strike. Proceedings of the Institution of Mechanical Engineers, 204, 21-28.

Jorgensen, U., \& Bojsen-Moller, F. (1989). Shock absobency of factors in the shoe/heel interaction - with special focus on role of the heel pad. Foot \& Ankle 9(11), 294-299.

Jorgensen, U., \& Ekstrand, J. (1988). Significance of heel pad confinement for the shock absorption at heel strike. International Journal of Sports Medicine, 9, 468-473. 
URL www.una.ac.cr/mhsalud

Keller, T. S., Weisberger, A. M., Ray, J. L., Hasan, S. S., Shiavi, R. G., \& Spengler, D. M. (1996). Relationship between vertical ground reaction force and speed during walking, slow jogging, and running. Clinical Biomechanics(11), 253-259.

Lafortune, M. A., \& Henning, E. M. (1992). Cushioning properties of footwear during walking: accelerometer and force platform measurements. Clinical Biomechanics, 7, 181-184.

Lafortune, M. A., Lake, M., \& Hennig, E. M. (1996). Differential shock transmission response of the human body to impact severity and lower limb posture. Journal of Biomechanics, 29(12), 1531-1537.

Lake, M., \& Robinson, M. (2005). The 7th Symposium on Footwear Biomechanics. Paper presented at the Biomechanics of walking in different shoes: a comparison between overground and treadmill testing protocols, Cleveland, Ohio, USA.

Lequesne, M. G., Dang, N., \& Lane, N. E. (1997). Sport practice and osteoarthritis of the limbs. Osteoarthritis Cartilage, 5(2), 75-86.

Light, L. H. (1979). Potential implications of heel strike transients. Journal of Physiology, 292, 31-32.

Light, L. H., MacLellan, G. E., \& Klenerman, L. (1980). Skeletal transients on heel strike in normal walking with different footwear. Journal of Biomechanics, 13, 477-480.

Martin, P. E., \& Marsh, A. P. (1992). Step length and frequency effects on ground reaction forces during walking. Technical note. Journal of Biomechanics, 25(10), 1237-1239.

McCaw, S. T., Heil, M. E., \& Hamill, J. (2000). The effect of comments about shoe construction on impact forces during walking. Medicine \& Science of Sport \& Exercise, 32(7), 1258-1164.

Menz, H. B., Latt, M. D., Tiedemann, A., Mun San Kwan, M., \& Lord, S. R. (2004). Reliability of the Gaitrite walkway system for the quantification of temporo-spatial parameters of gait in young and older people. Gait \& Posture, 20, 20-25.

Menz, H. B., Lord, S. R., \& Fitzpatrick, R. C. (2003). Age-Related Differences in Walking Stability. Age and Ageing, 32(2), 137-142.

Mercer, J. A., \& Vance, J. (2002, May). Spring-boots can reduce impact in runners. Biomechanics.

Milgrom, C., Giladi, M., Kashtan, H., Simkin, A., Chisin, R., Marguiles, J. (1985). A prospective study of a shock absorbing arthrotic device on the incidence of stress fractures in military recruits. Foot and Ankle, 6, 101-104.

Mills, P. M., \& Barrett, R. S. (2001). Swing Phase Mechanics of Healthy Young and Elderly Men. Human Movement Science, 20, 427-446.

Murray, M. P., Kory, R. C., Clarkson, B. H., \& Sepic, S. B. (1966). Comparison of free and fast speed walking patterns of normal men. American Journal of Physical Medicine \& Rehabilitation, 45(1), 8-24. 
URL www.una.ac.cr/mhsalud

Nigg, B., Cole, G., \& Bruggemann, P. (1995). Impact forces during heel-toe running. Journal of Applied Biomechanics, 11, 407-432.

Oeffinger, D., Brauch, B., Cranfill, S., Hisle, C., Wynn, C., Hicks, R. (1999). Comparison of gait with and without shoes in children. Gait \& Posture, 9, 95-100.

Pardo Merino, A., \& Ruiz Díaz, M. Á. (2002). SPSS 11. Guía para el análisis de datos. Madrid: Mc Graw Hill.

Payne, C., Zammitt, G., \& Patience, D. (2005). 7th Symposium on Footwear Biomechanics. Paper presented at the Predictors of a Response to Windlass Mechanism Enhancing Running Shoes, Cleveland, Ohio, USA.

Perry, J. (1992). Gait Analysis. Normal and Pathological Function. Yorba Linda, CA.: Slack incorporated.

Pollo, F. E., Gowling, T. L., \& Jackson, R. W. (1999). Walking boot design: a gait analysis study. Orthopedics, 22(5), 503-507.

Pratt, D. J., Rees, P. H., \& Rodgers, C. (1986). Assessment of some shock absorbing insoles (technical note). Prosthetics and Orthotics International, 10, 43-45.

Radin, E. L., Paul, I. L., \& Rose, R. M. (1972). Role of mechanical factors in the pathogenesis of primary osteoarthritis Lancet, 2, 519-522.

Sánchez Lacuesta, J. J., J.M., P. P., Hoyos Fuentes, J. V., Viosca Herreo, E., Soler Gracia, C., Comín Clavijo, M. (1999). Biomecánica de la Marcha Humana y Patológica (1 ed.). Valencia: Instituto de Biomecánica de Valencia (IBV).

Sloss, R. (2002). The effects of foot orthoses on othe ground reaction forces during walking. Part 1. The Foot, 11, 205-214.

Steinbach, H. L., \& Russel, W. (1964). Measurement of the heel pad as an aid to diagnosis of acromegaly. Radiology, 82, 418-423.

Tilbury-Davis, D. C., \& Hooper, R. H. (1999). The kinetic and kinematic effects of increasing load carriage upon the lower limb. Human Movement Science, 18, 693-700.

Verdini, F., Leo, T., Fioretti, S., Benedetti, M. G., Catani, F., \& Giannini, S. (2000). Analysis of ground reaction forces by means of wavelet transform. Clinical Biomechanics, 15, 607610.

Voloshin, A., \& Wosk, A. (1982). An In Vivo Study of Low back Pain and Shock Absorption in the Human Locomotor System. Journal of Biomechanics, 15(1), 21-27.

Voloshin, A. S., \& Wosk, A. (1981). Influence of artificial shock absorbers on human gait. Clinical Orthopaedics, 160, 52-56.

Vrezas, I., Elsner, G., Bolm-Audorff, U., Abolmaali, N., \& Seidler, A. (2010). Case-control study of knee osteoarthritis and lifestyle factors considering their interaction with physical workload. International Archives of Occupational and Environmental Health, 83(3), 291-300. doi: 10.1007/s00420-009-0486-6. 
URL www.una.ac.cr/mhsalud

Wakeling, J. M., Liphardt, A. M., \& Nigg, B. M. (2003). Muscle activity reduces soft-tissue resonance at heel-strike during walking. Journal of Biomechanics(36), 1761-1769.

Wakeling, J. M., Tscharner, V. V., Nigg, B. M., \& Stergiou, P. (2001). Muscle activity in the leg is tuned is response to ground reaction forces. Journal of Applied Physiology(91), 1307-1317.

Wang, C. L., Shau, Y. W., Hsu, T. C., Chen, H. C., \& Chien, S. H. (1999). Mechanical properties of heel pads reconstructed with flaps. The Journal of Bone And Joint Surgery, $81-B(2)$, 207-211.

Wearing, S. C., Urry, S. R., \& Smeathers, J. E. (2000). The Effect of Visual Targeting on Ground Reaction Force and Temporospatial Parameters of Gait. Clinical Biomechanics, 15, 583-591.

Whittle, M. W. (1997). Three-dimensional motion of the center of gravity of the body during walking. Human Movement Science, 16, 347-355.

Whittle, M. W. (1999). Generation and attenuation of transient impulsive forces beneath the foot: a review. Gait \& Posture(10), 264-275.

Windle, C. M., Gregory, S. M., \& Dixon, S. J. (1999). The shock attenuation characteristics of four different insoles when worn in a military boot during running and marching. Gait \& Posture(9), 31-37.

Fecha de recepción: 11 de agosto del 2011.

Fecha de aceptación: 11 de noviembre del 2011.

Fecha de publicación: 31 de diciembre del 2011. 